Submitted to the Annals of Statistics

arXiv: arXiv:1312.2645

\title{
SUPPLEMENT TO SUBSAMPLING BOOTSTRAP OF COUNT FEATURES OF NETWORKS
}

By Sharmodeep Bhattacharyya, ${ }^{*}$ and Peter J. Bickel*

University of California, Berkeley*

Oregon State University ${ }^{\dagger}$

\section{Appendix.}

A1. Variance of $\hat{P}_{b 1}(R)$. The variance of $\hat{P}_{b 1}(R)$ is

$$
\begin{aligned}
\operatorname{Var}_{b}\left[\frac{1}{\left(\begin{array}{c}
m \\
p
\end{array}\right)|I s o(R)|} \sum_{S \subseteq K_{m}, S \cong R} \mathbf{1}(S \subseteq H) \mid G\right] \\
=\left(\frac{1}{\left(\begin{array}{c}
m \\
p
\end{array}\right)|I s o(R)|}\right)^{2} \operatorname{Var}_{b}\left[\sum_{S \subseteq K_{m}, S \cong R} \mathbf{1}(S \subseteq H) \mid G\right]
\end{aligned}
$$

$\operatorname{Var}_{b}\left[\sum_{S \subseteq K_{m}, S \cong R} \mathbf{1}(S \subseteq H) \mid G\right]=\mathbb{E}_{b}\left[\left(\sum_{S \subseteq K_{m}, S \subseteq R} \mathbf{1}(S \subseteq H)\right)^{2} \mid G\right]$

$$
-\left(\mathbb{E}_{b}\left[\sum_{S \subseteq K_{m}, S \cong R} \mathbf{1}(S \subseteq H) \mid G\right]\right)^{2}
$$

$\mathbb{E}_{b}\left[\left(\sum_{S \subseteq K_{m}, S \cong R} \mathbf{1}(S \subseteq H)\right)^{2} \mid G\right]=\mathbb{E}_{b}\left[\sum_{S \subseteq K_{m}, S \cong R} \mathbf{1}(S \subseteq H) \mid G\right]$

$$
\begin{aligned}
& +\mathbb{E}_{b}\left[\sum_{\substack{S, T \subseteq K_{m} \\
S, T \cong \bar{R}, S \neq T}} \mathbf{1}(S, T \subseteq H) \mid G\right] \\
& =I+I I \text { (Suppose) }
\end{aligned}
$$

Thus,

$$
I=\sum_{S \subseteq K_{n}, S \cong R} \frac{\left(\begin{array}{c}
n-p \\
m-p
\end{array}\right)}{\left(\begin{array}{c}
n \\
m
\end{array}\right)} \mathbf{1}(S \subseteq G)
$$




$$
I I=\mathbb{E}_{b}\left[\sum_{\substack{S, T \subseteq K_{m} \\ S, T \cong \bar{R}, S \neq T}} \mathbf{1}(S, T \subseteq H) \mid G\right]
$$

Now, a host of subgraphs can be formed by the intersection of two copies of $R$. The number of intersected vertices can range from 0 to $p-1$. Let us consider, that for number of vertices in intersection as $k(k=1, \ldots,(p-1))$, the number of graph structures that can be formed is $g_{k}$ and we represent that graph structure by $W_{j k}$, where, $j=1, \ldots, g_{k}$. Thus,

$$
I I=\sum_{k=0}^{p-1} \sum_{j=1}^{g_{k}} \sum_{S \subseteq K_{n}, S \cong W_{j k}} \frac{\left(\begin{array}{c}
n-(2 p-k) \\
m-(2 p-k)
\end{array}\right)}{\left(\begin{array}{l}
n \\
m
\end{array}\right)} \mathbf{1}(S \subseteq G)
$$

So,

$$
\begin{aligned}
\mathbb{E}_{b}\left[\left(\sum_{S \subseteq K_{m}, S \cong R} \mathbf{1}(S \subseteq H)\right)^{2} \mid G\right]= & \sum_{S \subseteq K_{n}, S \cong R} \frac{\left(\begin{array}{c}
n-p \\
m-p
\end{array}\right)}{\left(\begin{array}{l}
n \\
m
\end{array}\right)} \mathbf{1}(S \subseteq G) \\
+ & \sum_{k=0}^{p-1} \sum_{j=1}^{g_{k}} \\
& \sum_{S \subseteq K_{n}, S \cong W_{j k}} \frac{\left(\begin{array}{c}
n-(2 p-k) \\
m-(2 p-k)
\end{array}\right)}{\left(\begin{array}{l}
n \\
m
\end{array}\right)} \mathbf{1}(S \subseteq G)
\end{aligned}
$$

$$
\begin{aligned}
\operatorname{Var}_{b}\left[\sum_{S \subseteq K_{m}, S \subseteq R} \mathbf{1}(S \subseteq H) \mid G\right]= & \sum_{S \subseteq K_{n}, S \cong R} \frac{\left(\begin{array}{c}
n-p \\
m-p
\end{array}\right)}{\left(\begin{array}{l}
n \\
m
\end{array}\right)} \mathbf{1}(S \subseteq G) \\
& +\sum_{k=0}^{p-1} \sum_{j=1}^{g_{k}} \\
& \sum_{S \subseteq K_{n}, S \subseteq W_{j k}} \frac{\left(\begin{array}{c}
n-(2 p-k) \\
m-(2 p-k)
\end{array}\right)}{\left(\begin{array}{l}
n \\
m
\end{array}\right)} \mathbf{1}(S \subseteq G) \\
& -\left(\sum_{S \subseteq K_{n}, S \cong R} \frac{\left(\begin{array}{c}
n-p \\
m-p
\end{array}\right)}{\left(\begin{array}{l}
n \\
m
\end{array}\right)} \mathbf{1}(S \subseteq G)\right)^{2}
\end{aligned}
$$




$$
\begin{aligned}
\operatorname{Var}_{b}\left[\frac{1}{\left(\begin{array}{c}
m \\
p
\end{array}\right)|I s o(R)|} \sum_{S \subseteq K_{m}, S \subseteq R} \mathbf{1}(S \subseteq H) \mid G\right] \\
=\left(\frac{1}{\left(\begin{array}{c}
m \\
p
\end{array}\right)|I s o(R)|}\right)^{2}\left[\sum_{S \subseteq K_{n}, S \cong R} \frac{\left(\begin{array}{c}
n-p \\
m-p
\end{array}\right)}{\left(\begin{array}{c}
n \\
m
\end{array}\right)} \mathbf{1}(S \subseteq G)\right] \\
\left.-\left(\frac{1}{\left(\begin{array}{c}
m \\
p
\end{array}\right)|I s o(R)|}\right)^{2}\left[\sum_{S \subseteq K_{n}, S \cong R} \frac{\left(\begin{array}{c}
n-p \\
m-p
\end{array}\right)}{\left(\begin{array}{c}
n \\
m
\end{array}\right)} \mathbf{1}(S \subseteq G)\right)^{2}\right] \\
+\left(\frac{1}{\left(\begin{array}{c}
m \\
p
\end{array}\right)|I s o(R)|}\right)^{2}\left[\sum_{k=0}^{p-1} \sum_{j=1}^{g} \sum_{\substack{S \subseteq K_{n} \\
S \cong W_{j k}}} \frac{\left(\begin{array}{c}
n-(2 p-k) \\
m-(2 p-k)
\end{array}\right)}{\left(\begin{array}{c}
n \\
m
\end{array}\right)} \mathbf{1}(S \subseteq G)\right]
\end{aligned}
$$

So,

$$
\begin{aligned}
\operatorname{Var}_{b}\left[\hat{P}_{b 1}(R)\right] & =\left(\frac{1}{\left(\begin{array}{l}
m \\
p
\end{array}\right)|I s o(R)|}\right)^{2}\left[\sum_{S \subseteq K_{n}, S \subseteq R} \frac{\left(\begin{array}{c}
n-p \\
m-p
\end{array}\right)}{\left(\begin{array}{c}
n \\
m
\end{array}\right)} \mathbf{1}(S \subseteq G)\right] \\
& -\left(\frac{1}{\left(\begin{array}{c}
m \\
p
\end{array}\right)|I s o(R)|}\right)^{2}\left[\left(\sum_{S \subseteq K_{n}, S \cong R} \frac{\left(\begin{array}{c}
n-p \\
m-p
\end{array}\right)}{\left(\begin{array}{l}
n \\
m
\end{array}\right)} \mathbf{1}(S \subseteq G)\right)^{2}\right] \\
& +\left(\frac{1}{\left(\begin{array}{c}
m \\
p
\end{array}\right)|I s o(R)|}\right)^{2}\left[\sum_{k=0}^{p-1} \sum_{j=1}^{g_{k}} \sum_{\substack{S \subseteq K_{n} \\
S \subseteq W_{j k}}} \frac{\left(\begin{array}{c}
n-(2 p-k) \\
m-(2 p-k)
\end{array}\right)}{\left(\begin{array}{l}
n \\
m
\end{array}\right)} \mathbf{1}(S \subseteq G)\right]
\end{aligned}
$$

A2. Proof of Theorem 3.1.

Proof. (i) Now, let us try to try to find the expectation of $\hat{P}_{b 1}(R)$ 
under the sampling distribution conditional on the given data $G$.

$$
\begin{array}{r}
\mathbb{E}_{b}\left[\frac{1}{\left(\begin{array}{c}
m \\
p
\end{array}\right)|I s o(R)|} \sum_{S \subseteq K_{m}, S \cong R} \mathbf{1}(S \subseteq H) \mid G\right] \\
=\frac{1}{\left(\begin{array}{c}
m \\
p
\end{array}\right)|I s o(R)|} \mathbb{E}\left[\sum_{S \subseteq K_{m}, S \cong R} \mathbf{1}(S \subseteq H) \mid G\right] \\
=\frac{1}{\left(\begin{array}{c}
m \\
p
\end{array}\right)|I s o(R)|} \sum_{H \subseteq G,|H|=m} \frac{1}{\left(\begin{array}{l}
n \\
m
\end{array}\right)} \sum_{S \subseteq K_{m}, S \cong R} \mathbf{1}(S \subseteq H) \\
=\frac{1}{\left(\begin{array}{c}
m \\
p
\end{array}\right)|I s o(R)|} \sum_{\substack{S \subseteq K_{n} \\
S \subseteq R}} \sum_{\substack{|H, H \subseteq G\\
| H \mid=m}} \frac{1}{\left(\begin{array}{c}
n \\
m
\end{array}\right)} \mathbf{1}(S \subseteq G) \\
=\frac{1}{\left(\begin{array}{c}
m \\
p
\end{array}\right)|I s o(R)|} \sum_{S \subseteq K_{n}, S \cong R} \frac{\left(\begin{array}{l}
n-p \\
m-p
\end{array}\right)}{\left(\begin{array}{c}
n \\
m
\end{array}\right)} \mathbf{1}(S \subseteq G) \\
=\frac{1}{\left(\begin{array}{l}
n \\
p
\end{array}\right)|I s o(R)|} \sum_{S \subseteq K_{n}, S \cong R} \mathbf{1}(S \subseteq G)
\end{array}
$$

So, we have,

$$
\mathbb{E}_{b}\left[\bar{P}_{B 1}(R) \mid G\right]=\mathbb{E}_{b}\left[\hat{P}_{b 1}(R) \mid G\right]=\hat{P}(R)
$$

(ii) Given $G$,

$$
\begin{aligned}
\operatorname{Var}_{b}\left[\rho^{-e} \bar{P}_{B 1}(R) \mid G\right]=\rho^{-2 e} \frac{1}{B^{2}} & \left(\sum_{b=1}^{B} \operatorname{Var}_{b}\left[\hat{P}_{b 1}(R)\right]\right. \\
& \left.+\sum_{b, b^{\prime}=1, b \neq b^{\prime}}^{B} \operatorname{Cov}_{b}\left(\hat{P}_{b 1}(R), \hat{P}_{b^{\prime} 1}(R)\right)\right)
\end{aligned}
$$

Now, the formula for $\operatorname{Var}_{b}\left[\hat{P}_{b 1}(R)\right]$ from A1 we get that

$$
\begin{aligned}
\operatorname{Var}_{b}\left[\rho_{n}^{-e} \hat{P}_{b 1}(R)\right] & =\left(\frac{\rho_{n}^{-e}}{\left(\begin{array}{l}
m \\
p
\end{array}\right)|I s o(R)|}\right)^{2}\left[\sum_{S \subseteq K_{n}, S \cong R} \frac{\left(\begin{array}{c}
n-p \\
m-p
\end{array}\right)}{\left(\begin{array}{l}
n \\
m
\end{array}\right)} \mathbf{1}(S \subseteq G)\right] \\
& -\left(\frac{\rho_{n}^{-e}}{\left(\begin{array}{c}
m \\
p
\end{array}\right)|I s o(R)|}\right)^{2}\left[\left(\sum_{S \subseteq K_{n}, S \cong R} \frac{\left(\begin{array}{c}
n-p \\
m-p
\end{array}\right)}{\left(\begin{array}{l}
n \\
m
\end{array}\right)} \mathbf{1}(S \subseteq G)\right)^{2}\right] \\
& +\left(\frac{\rho_{n}^{-e}}{\left(\begin{array}{c}
m \\
p
\end{array}\right)|I s o(R)|}\right)^{2}\left[\sum_{k=0}^{p-1} \sum_{j=1}^{g_{k}} \sum_{\substack{S \subseteq K_{n} \\
S \cong W_{j k}}} \frac{\left(\begin{array}{c}
n-(2 p-k) \\
m-(2 p-k)
\end{array}\right)}{\left(\begin{array}{c}
n \\
m
\end{array}\right)} \mathbf{1}(S \subseteq G)\right]
\end{aligned}
$$


So,

$$
\begin{aligned}
\operatorname{Var}_{b}\left[\rho_{n}^{-e} \hat{P}_{b 1}(R)\right] & =O\left(\frac{1}{\rho_{n}^{e}\left(\begin{array}{c}
m \\
p
\end{array}\right)}\right)+O\left(\frac{\left(\begin{array}{c}
m \\
p_{W}
\end{array}\right) \rho_{n}^{e_{W}}}{\left(\begin{array}{c}
m \\
p
\end{array}\right)^{2} \rho_{n}^{2 e}}\right) \\
& =O\left(\frac{1}{m^{p} \rho_{n}^{e}}\right)+O\left(\frac{1}{m}\right)
\end{aligned}
$$

where, $W=S \cup S^{\prime}, p_{W}=|V(W)|$ and $e_{W}=|E(W)|$.

(iii) Here, we use properties of the underlying model. Let us condition on $\boldsymbol{\xi}=\left\{\xi_{1}, \ldots, \xi_{n}\right\}$ and the whole graph $G$ separately. Now, conditioning on $\boldsymbol{\xi}$, we get the main term of $\hat{P}(R)$ to be,

$\mathbb{E}\left(\rho^{-e} \hat{P}(R) \mid \boldsymbol{\xi}\right)=\frac{1}{\left(\begin{array}{l}n \\ p\end{array}\right)|I s o(R)|} \sum_{S \subseteq K_{n}, S \cong R}\left(\prod_{(i, j) \in E(S)} w\left(\xi_{i}, \xi_{j}\right)\right)+O\left(n^{-1} \lambda_{n}\right)$.

We shall use the same decomposition as used in [1] of $\left(\rho_{n}^{-e} \bar{P}_{B 1}(R)-\right.$ $\tilde{P}(R))$ into

$$
\begin{aligned}
\left(\rho_{n}^{-e} \bar{P}_{B 1}(R)-\tilde{P}(R)\right)= & \rho_{n}^{-e}\left(\bar{P}_{B 1}-\mathbb{E}_{b}\left[\hat{P}_{b 1}(R) \mid G\right]\right) \\
& +\rho_{n}^{-e}(\hat{P}(R)-\mathbb{E}(\hat{P}(R) \mid \boldsymbol{\xi})) \\
& +\mathbb{E}(\hat{P}(R) \mid \boldsymbol{\xi}) \rho_{n}^{-e}-\tilde{P}(R)
\end{aligned}
$$

Let us define,

$$
\begin{aligned}
U_{3} & =\mathbb{E}(\hat{P}(R) \mid \boldsymbol{\xi}) \rho_{n}^{-e}-\tilde{P}(R) \\
U_{2} & =\rho_{n}^{-e}(\hat{P}(R)-\mathbb{E}(\hat{P}(R) \mid \boldsymbol{\xi})) \\
U_{1} & =\rho_{n}^{-e}\left(\bar{P}_{B 1}-\mathbb{E}_{b}\left[\hat{P}_{b 1}(R) \mid G\right]\right)
\end{aligned}
$$

Now, it is easy to see that

$$
\begin{aligned}
\operatorname{Var}\left(\rho^{-e} \bar{P}_{B 1}(R)\right) & =\mathbb{E}\left(\operatorname{Var}\left(\rho^{-e} \bar{P}_{B 1}(R) \mid G\right)\right)+\operatorname{Var}\left(\mathbb{E}\left(\rho^{-e} \bar{P}_{B 1}(R) \mid G\right)\right) \\
& =\mathbb{E}\left(\operatorname{Var}\left(\rho^{-e} \bar{P}_{B 1}(R)-\hat{P}(R) \mid G\right)+\operatorname{Var}(\hat{P}(R))\right. \\
& =\mathbb{E}\left(\operatorname{Var}\left(U_{1} \mid G\right)\right)+\mathbb{E}(\operatorname{Var}(\hat{P}(R) \mid \boldsymbol{\xi}))+\operatorname{Var}(\mathbb{E}(\hat{P}(R) \mid \boldsymbol{\xi})) \\
& =\mathbb{E}\left(\operatorname{Var}\left(U_{1} \mid G\right)\right)+\mathbb{E}\left(\operatorname{Var}\left(U_{2} \mid \boldsymbol{\xi}\right)\right)+\operatorname{Var}\left(U_{3}\right)
\end{aligned}
$$

We shall try to see the behavior of $\operatorname{Var}\left(U_{1} \mid G\right)=\operatorname{Var}_{b}\left[\rho^{-e} \bar{P}_{B 1}(R) \mid G\right]$. From (ii) we get that, $\operatorname{Var}_{b}\left[\rho_{n}^{-e} \hat{P}_{b 1}(R)\right]=O\left(\frac{1}{m^{p} \rho_{n}^{e}} \vee \frac{1}{m}\right)$. Similarly, $\operatorname{Cov}_{b}\left[\rho_{n}^{-e} \hat{P}_{b 1}(R), \rho_{n}^{-e} \hat{P}_{b^{\prime} 1}(R)\right]=O\left(\frac{1}{m}\right)$ for acyclic and $k$-cycle $R$ following similar steps as variance in Appendix A1. If we consider the uniform probability for bootstrap to be $\gamma$, then, $B=O\left(\gamma n^{p}\right)$. Note that, 
if $E\left(H_{b}\right) \cap E\left(H_{b^{\prime}}\right)=\phi$, then, $\operatorname{Cov}_{b}\left(\hat{P}_{b 1}(R), \hat{P}_{b^{\prime} 1}(R)\right)=0$. The number of pairs such that $E\left(H_{b}\right) \cap E\left(H_{b^{\prime}}\right) \neq \phi$ is $O\left(m^{2} \gamma^{2} n^{2 m-2}\right)$. Also, the number of edges for the leading term in the covariance is equal to or more than $2 e$. So,

$$
\begin{array}{r}
\mathbb{E}\left(\operatorname{Var}_{b}\left[\rho^{-e} \bar{P}_{B 1}(R) \mid G\right]\right)=O\left(\frac{1}{B\left(m^{p} \rho_{n}^{e} \wedge m\right)}\right)+O\left(\frac{m^{2} \gamma^{2} n^{2 m-2}}{m \gamma^{2} n^{2 m}}\right) \\
\quad=O\left(\frac{1}{B\left(m^{p} \rho_{n}^{e} \wedge m\right)}+\frac{m}{n^{2}}\right)=O\left(\frac{1}{B\left(m^{p} \rho_{n}^{e} \wedge m\right)}\right)+o\left(n^{-1}\right)
\end{array}
$$

The second equality follows since we have $m / n \rightarrow 0$ as $n \rightarrow \infty$. So, since, $B\left(m^{p} \rho_{n}^{e} \wedge m\right)>O(n)$, we have, $\mathbb{E}\left(\operatorname{Var}\left(U_{1} \mid G\right)\right)=o\left(n^{-1}\right)$.

Now, by proof of Theorem 1 in [1], we have,

$$
\begin{aligned}
& \operatorname{Var}\left(U_{2}\right)=o\left(n^{-1}\right) \\
& \operatorname{Var}\left(U_{3}\right)=o\left(n^{-1}\right)
\end{aligned}
$$

So, we get, $\operatorname{Var}\left(\rho^{-e} \bar{P}_{B 1}(R)\right)=o\left(n^{-1}\right)$. Since, we already know $\sqrt{n}$ consistency of $\left.\left(\rho_{n}^{-e} \hat{P}(R)-\tilde{P}(R)\right)\right)$, this proves the $\sqrt{n}$-consistency of $\rho_{n}^{-e} \bar{P}_{B 1}(R)$ to $\rho_{n}^{-e} \hat{P}(R)$.

A3. Proof of Theorem 3.2. For variance calculation, we also need the joint inclusion probability of two items, $S, S^{\prime} \in \mathcal{S}_{p}$, which are subgraphs of $G$ induced by the set of vertices $\left\{w_{1}, \ldots, w_{p}\right\}$ and $\left\{w_{1}^{\prime}, \ldots, w_{p}^{\prime}\right\}$ respectively, where, we take that $w_{i+1} \succ w_{i}$ and $w_{i+1}^{\prime} \succ w_{i}^{\prime}, i=1, \ldots, p-1$. So,

$\pi_{S S^{\prime}} \equiv$ Inclusion Probability of $S$ and $S^{\prime}=\mathbb{P}\left[\left(w_{1}, \ldots, w_{p}\right)\right.$ is selected and $\left(w_{1}^{\prime}, \ldots, w_{p}^{\prime}\right)$ is selected]

$$
=\prod_{d=1}^{p}\left(q_{d}\right)^{z_{1 d}} \prod_{d=1}^{p}\left(q_{d}^{2}\right)^{z_{2 d}}
$$

where,

$$
\begin{aligned}
& z_{1 d}= \begin{cases}\mathbf{1}\left(w_{d}=w_{d}^{\prime}\right), & \text { for } d=1 \\
\mathbf{1}\left(\left(w_{d}, w_{d-1}\right)=\left(w_{d}^{\prime}, w_{d-1}^{\prime}\right)\right), & \text { for } d=2, \ldots, p\end{cases} \\
& z_{2 d}= \begin{cases}\mathbf{1}\left(w_{d} \neq w_{d}^{\prime}\right), & \text { for } d=1 \\
\mathbf{1}\left(\left(w_{d}, w_{d-1}\right) \neq\left(w_{d}^{\prime}, w_{d-1}^{\prime}\right)\right), & \text { for } d=2, \ldots, p\end{cases}
\end{aligned}
$$


(i) We know that $\hat{P}_{b 2}(R)$ is a Horvitz-Thompson estimator with inclusion probability of each population unit to be $\pi=\prod_{d=1}^{p} q_{d}$. So, according to the sampling theory [2], $\hat{P}_{b 2}(R)$ is an unbiased estimator of $\hat{P}(R)$ given the network $G$, if $\mathbb{P}\left(\hat{P}_{b 2}(R)=0 \mid \hat{P}(R)\right) \rightarrow 0$ as $n \rightarrow \infty$. Now, $\mathbb{P}\left(\hat{P}_{b 2}(R)=0 \mid \hat{P}(R)\right) \leq\left(1-q_{d}\right)^{\lambda_{n}}$ for all $d=1, \ldots, p$. For all $d=$ $1, \ldots, p,\left(1-q_{d}\right)^{\lambda_{n}} \rightarrow 0$ if $\lambda_{n} q_{d} \rightarrow \infty$. So, under the condition, $\lambda_{n} q_{d} \infty$ and $q_{d} \rightarrow 0$ as $n \rightarrow \infty$, we have, $\hat{P}_{b 2}(R)$ is an asymptotically unbiased estimator of $\hat{P}(R)$.

(ii) The variance of $\hat{P}_{b 2}(R)$ coming from the bootstrap sampling only is given by

$$
\begin{aligned}
\operatorname{Var}_{b}\left[\rho_{n}^{-e} \hat{P}_{b 2}(R)\right]= & \frac{1}{N^{2}}\left[\frac{1-\pi}{\pi} \sum_{S \in \mathcal{S}_{p}} \mathbf{1}(S \cong R)\right. \\
& \left.+\sum_{S, S^{\prime} \in \mathcal{S}_{p}, S \neq S^{\prime}} \frac{\pi_{S S^{\prime}}-\pi^{2}}{\pi^{2}} \mathbf{1}\left(S \cong R, S^{\prime} \cong R\right)\right]
\end{aligned}
$$

where,

$$
N=\rho_{n}^{e}\left(\begin{array}{l}
n \\
p
\end{array}\right)|I s o(R)|
$$

From the formula of $\operatorname{Var}_{b}\left[\hat{P}_{b 2}(R) \mid G\right]$, we see that, the covariance terms vanishes when $\pi_{S S^{\prime}}=\pi^{2}$. Now, if $q_{1}=1$, then, $\pi_{S S^{\prime}}=\pi^{2}$ if $E(S) \cap$ $E\left(S^{\prime}\right)=\phi$. The number of pairs such that $E(S) \cap E\left(S^{\prime}\right) \neq \phi$ is $O\left(p^{2} n^{2 p-2}\right)$.

Now, the condition of $q_{1}=1$ is a bit restrictive. In stead, if we have $q_{1} \rightarrow 1$ as $n \rightarrow \infty$, then, the highest order term of covariance term comes from the case when $E(S) \cap E\left(S^{\prime}\right) \neq \phi$ but the root nodes are same that is $w_{1}=w_{1}^{\prime}$. So, for some constant $C>0$,

$$
\begin{array}{r}
\frac{1}{N^{2}} \sum_{S, S^{\prime} \in \mathcal{S}_{p}, S \neq S^{\prime}} \frac{\pi_{S S^{\prime}}-\pi^{2}}{\pi^{2}} \mathbf{1}\left(S \cong R, S^{\prime} \cong R\right) \\
\leq \frac{C}{N^{2}} \sum_{S, S^{\prime} \in \mathcal{S}_{p}, S \neq S^{\prime}} \frac{q_{1}-q_{1}^{2}}{q_{1}^{2}} \mathbf{1}\left(S \cong R, S^{\prime} \cong R\right) \\
=O\left(\left(\frac{1}{q_{1}}-1\right) \frac{n^{2 p-1}}{n^{2 p}}\right) \\
=O\left(\left(\frac{1}{q_{1}}-1\right) \frac{1}{n}\right)
\end{array}
$$


Now, for the variance term to vanish we need the conditions $q_{1}=1$ or $q_{1} \rightarrow 1$ and $q_{d} \rightarrow 0$ and $\lambda_{n} q_{d} \rightarrow \infty$ for $d=2, \ldots, p$ as $n \rightarrow \infty$. Since, we know that $O(1) \leq \lambda_{n} O(n)$, we get $n q_{d} \rightarrow \infty$ for $d=2, \ldots, p$ as $n \rightarrow \infty$. So, we have

$$
\begin{aligned}
\frac{1}{N^{2}} \frac{1-\pi}{\pi} \sum_{S \in \mathcal{S}_{p}} \mathbf{1}(S \cong R) & =\left(\frac{1}{\pi}-1\right) O\left(\frac{1}{n^{p} \rho^{e}}\right) \\
& =O\left(\frac{1}{n^{p} \rho_{n}^{e} \pi}\right) \\
& =O\left(\frac{1}{n \rho_{n}^{e-p+1}} \cdot \prod_{d=2}^{p} \frac{1}{\lambda_{n} q_{d}}\right)
\end{aligned}
$$

So,

$$
\mathbb{E}\left(\operatorname{Var}_{b}\left[\rho_{n}^{-e} \hat{P}_{b 2}(R)\right]\right)=O\left(\left(\frac{1}{q_{1}}-1\right) \frac{1}{n}\right)+O\left(\frac{1}{n \rho_{n}^{e-p+1}} \cdot \prod_{d=2}^{p} \frac{1}{\lambda_{n} q_{d}}\right)
$$

(iii) We shall use the same decomposition as used in [1] of $\left(\rho_{n}^{-e} \bar{P}_{B 2}(R)-\right.$ $\tilde{P}(R))$ into

$$
\begin{aligned}
\left(\rho_{n}^{-e} \bar{P}_{B 2}(R)-\tilde{P}(R)\right)= & \rho_{n}^{-e}\left(\bar{P}_{B 2}-\mathbb{E}_{b}\left[\hat{P}_{b 2}(R) \mid G\right]\right) \\
& +\rho_{n}^{-e}(\hat{P}(R)-\mathbb{E}(\hat{P}(R) \mid \boldsymbol{\xi})) \\
& +\mathbb{E}(\hat{P}(R) \mid \boldsymbol{\xi}) \rho_{n}^{-e}-\tilde{P}(R)
\end{aligned}
$$

Let us define,

$$
\begin{aligned}
U_{3} & =\mathbb{E}(\hat{P}(R) \mid \boldsymbol{\xi}) \rho_{n}^{-e}-\tilde{P}(R) \\
U_{2} & =\rho_{n}^{-e}(\hat{P}(R)-\mathbb{E}(\hat{P}(R) \mid \boldsymbol{\xi})) \\
U_{1} & =\rho_{n}^{-e}\left(\bar{P}_{B 2}-\mathbb{E}_{b}[\hat{P}(R) \mid G]\right)
\end{aligned}
$$

Now, it is easy to see that

$$
\begin{aligned}
\operatorname{Var}\left(\rho^{-e} \bar{P}_{B 2}(R)\right) & =\mathbb{E}\left(\operatorname{Var}\left(\rho^{-e} \bar{P}_{B 2}(R) \mid G\right)\right)+\operatorname{Var}\left(\mathbb{E}\left(\rho^{-e} \bar{P}_{B 2}(R) \mid G\right)\right) \\
& =\mathbb{E}\left(\operatorname{Var}\left(\rho^{-e} \bar{P}_{B 2}(R)-\hat{P}(R) \mid G\right)+\operatorname{Var}(\hat{P}(R))\right. \\
& =\mathbb{E}\left(\operatorname{Var}\left(U_{1} \mid G\right)\right)+\mathbb{E}(\operatorname{Var}(\hat{P}(R) \mid \boldsymbol{\xi}))+\operatorname{Var}(\mathbb{E}(\hat{P}(R) \mid \boldsymbol{\xi})) \\
& =\mathbb{E}\left(\operatorname{Var}\left(U_{1} \mid G\right)\right)+\mathbb{E}\left(\operatorname{Var}\left(U_{2} \mid \boldsymbol{\xi}\right)\right)+\operatorname{Var}\left(U_{3}\right)
\end{aligned}
$$

We shall try to see the behavior of $\operatorname{Var}_{b}\left[\rho_{n}^{-e} \hat{P}_{b 2}(R) \mid G\right]$.

$$
\mathbb{E}\left(\operatorname{Var}_{b}\left[\rho_{n}^{-e} \hat{P}_{b 2}(R)\right]\right)=O\left(\left(\frac{1}{q_{1}}-1\right) \frac{1}{n}\right)+O\left(\frac{1}{n \rho_{n}^{e-p+1}} \cdot \prod_{d=2}^{p} \frac{1}{\lambda_{n} q_{d}}\right)
$$


Now, since the bootstrap samples for subgraph sampling are selected independently, we have that,

$\mathbb{E}\left(\operatorname{Var}_{b}\left[\rho_{n}^{-e} \bar{P}_{B 2}(R)\right]\right)=O\left(\left(\frac{1}{q_{1}}-1\right) \frac{1}{n B}\right)+O\left(\frac{1}{B n \rho_{n}^{e-p+1}} \cdot \prod_{d=2}^{p} \frac{1}{\lambda_{n} q_{d}}\right)$

Now, under the condition $\frac{1}{B}\left(\frac{1}{q_{1}}-1\right) \rightarrow 0, q_{d} \rightarrow 0$ for all $d=1, \ldots, p$ and $B \prod_{d=2}^{p} q_{d} \geq \frac{1}{n^{p-1} \rho_{n}^{e}}$, we have

$$
\mathbb{E}\left(\operatorname{Var}\left(U_{1} \mid G\right)\right)=\mathbb{E}\left(\operatorname{Var}_{b}\left[\rho_{n}^{-e} \hat{P}_{b 2}(R) \mid G\right]\right)=o\left(n^{-1}\right)
$$

Now, by proof of Theorem 1 in [1], we have,

$$
\begin{aligned}
& \operatorname{Var}\left(U_{2}\right)=o\left(n^{-1}\right) \\
& \operatorname{Var}\left(U_{3}\right)=o\left(n^{-1}\right)
\end{aligned}
$$

So, we get, $\operatorname{Var}\left(\rho^{-e} \bar{P}_{B 2}(R)\right)=o\left(n^{-1}\right)$. Since, we already know $\sqrt{n}$ consistency of $\left.\left(\rho_{n}^{-e} \hat{P}(R)-\tilde{P}(R)\right)\right)$, this proves the $\sqrt{n}$-consistency of $\rho_{n}^{-e} \bar{P}_{B 2}(R)$ to $\rho_{n}^{-e} \hat{P}(R)$.

B1. Proof of Proposition 6.

$$
\begin{gathered}
\sigma^{2}(R ; \rho)=\operatorname{Var}\left[\rho^{-e} \hat{P}(R)\right] \\
=\operatorname{Var}\left[\sum_{S \subseteq K_{n}, S \subseteq R} \frac{\mathbf{1}(S \subseteq G)}{\rho^{e}\left(\begin{array}{l}
n \\
p
\end{array}\right)|I s o(R)|}\right] \\
=\frac{1}{\left(\rho^{e}\left(\begin{array}{l}
n \\
p
\end{array}\right)|I s o(R)|\right)^{2}} \mathbb{E}\left[\sum_{S \subseteq K_{n}, S \cong R} \mathbf{1}(S \subseteq G)\right]^{2}-(\tilde{P}(R))^{2} \\
=\frac{1}{\left(\rho^{e}\left(\begin{array}{c}
n \\
p
\end{array}\right)|I s o(R)|\right)^{2}} \mathbb{E}\left[\sum_{\substack{S, T \subseteq K_{n} \\
S, T \cong R, S \cap T \neq \emptyset}} \mathbf{1}(S, T \subseteq G)\right]-\left(1-\frac{\left(\begin{array}{c}
n-p \\
p
\end{array}\right)}{\left(\begin{array}{l}
n \\
p
\end{array}\right)}\right)(\tilde{P}(R))^{2}
\end{gathered}
$$

If $R$ is a connected subgraph, then, we can write,

$$
\begin{aligned}
\frac{1}{\left(\rho^{e}\left(\begin{array}{l}
n \\
p
\end{array}\right)|I s o(R)|\right)^{2}} \mathbb{E}\left[\sum_{\substack{S, T \subseteq K_{n} \\
S, T \cong R, S \cap T \neq \emptyset}} \mathbf{1}(S, T \subseteq G)\right] \\
=\frac{1}{\left(\rho^{e}\left(\begin{array}{l}
n \\
p
\end{array}\right)|I s o(R)|\right)^{2}} \sum_{\substack{W: W=S \cup T \\
S, T \cong R, S \cap T \neq \emptyset}} \mathbb{E}\left[\sum_{W \subseteq K_{n}} \mathbf{1}(W \subseteq G)\right]
\end{aligned}
$$




$$
\begin{aligned}
& \sigma\left(R_{1}, R_{2} ; \rho\right)=\operatorname{Cov}\left(\rho^{-e_{1}} \hat{P}\left(R_{1}\right), \rho^{-e_{2}} \hat{P}\left(R_{2}\right)\right) \\
& =\frac{1}{\left(\rho^{e_{1}+e_{2}}\left(\begin{array}{c}
n \\
p_{1}
\end{array}\right)\left(\begin{array}{c}
n \\
p_{2}
\end{array}\right)\left|I s o\left(R_{1}\right)\right|\left|I s o\left(R_{2}\right)\right|\right)} \times \\
& \mathbb{E}\left[\left(\sum_{\substack{S \subseteq K_{n} \\
S \cong R_{1}}} \mathbf{1}(S \subseteq G)\right)\left(\sum_{\substack{S \subseteq K_{n} \\
S \cong R_{2}}} \mathbf{1}(S \subseteq G)\right)\right]-\tilde{P}\left(R_{1}\right) \tilde{P}\left(R_{2}\right) \\
& =\frac{1}{\left(\rho^{e_{1}+e_{2}}\left(\begin{array}{c}
n \\
p_{1}
\end{array}\right)\left(\begin{array}{c}
n \\
p_{2}
\end{array}\right)\left|I s o\left(R_{1}\right)\right|\left|I s o\left(R_{2}\right)\right|\right)} \mathbb{E}\left[\sum_{\substack{S, T \subseteq K_{n} \\
S \cong R_{1}, T \cong R_{2}, S \cap T \neq \emptyset}} \mathbf{1}(S, T \subseteq G)\right] \\
& -\left(1-\frac{\left(\begin{array}{c}
n-p_{1} \\
p_{2}
\end{array}\right)}{\left(\begin{array}{c}
n \\
p_{2}
\end{array}\right)}\right) \tilde{P}\left(R_{1}\right) \tilde{P}\left(R_{2}\right)
\end{aligned}
$$

If $R$ is a connected subgraph, then, we can write,

$$
\begin{aligned}
& \frac{1}{\left(\rho^{e_{1}+e_{2}}\left(\begin{array}{c}
n \\
p_{1}
\end{array}\right)\left(\begin{array}{c}
n \\
p_{2}
\end{array}\right)\left|I s o\left(R_{1}\right)\right|\left|I s o\left(R_{2}\right)\right|\right)} \mathbb{E}\left[\sum_{\substack{S, T \subseteq K_{n} \\
S \cong R_{1}, T \cong R_{2}, S \cap T \neq \emptyset}} \mathbf{1}(S, T \subseteq G)\right] \\
= & \frac{1}{\left(\rho^{e_{1}+e_{2}}\left(\begin{array}{c}
n \\
p_{1}
\end{array}\right)\left(\begin{array}{c}
n \\
p_{2}
\end{array}\right)\left|I s o\left(R_{1}\right)\right|\left|I s o\left(R_{2}\right)\right|\right)} \sum_{\substack{W: W=S \cup T \\
S \cong R_{1} T \cong R_{2}, S \cap T \neq \emptyset}} \mathbb{E}\left[\sum_{W \subseteq K_{n}} \mathbf{1}(W \subseteq G)\right]
\end{aligned}
$$

B2. Proof of Lemma \%. Let us define

$$
\tilde{\sigma}^{2}(R)=\frac{1 /(1-x)}{\left(\rho_{n}^{e}\left(\begin{array}{l}
n \\
p
\end{array}\right)|I s o(R)|\right)^{2}} \sum_{\substack{W \subseteq K_{n}: W=S \cup T, S, T \cong R,|S \cap T|=1, p}} \mathbf{1}(W \subseteq G)-\frac{x \rho_{n}^{-2 e} \hat{P}(R)^{2}}{(1-x)}
$$

where, $x=\left(1-\frac{((n-p) !)^{2}}{n !(n-2 p) !}\right)$ and

$$
\begin{aligned}
\tilde{\sigma}\left(R_{1}, R_{2}\right)=\frac{1 /(1-y)}{\left(\rho_{n}^{e_{1}+e_{2}}\left(\begin{array}{c}
n \\
p_{1}
\end{array}\right)\left(\begin{array}{c}
n \\
p_{2}
\end{array}\right)\left|I s o\left(R_{1}\right)\right|\left|I s o\left(R_{2}\right)\right|\right)} \\
\sum_{\substack{W \subseteq K_{n}, W=S \cup T, S \cong R_{1}, T \cong R_{2},|S \cap T|=1}} \mathbf{1}(W \subseteq G)-\frac{y \rho_{n}^{-\left(e_{1}+e_{2}\right)} \hat{P}\left(R_{1}\right) \hat{P}\left(R_{2}\right)}{(1-y)} .
\end{aligned}
$$


where, $y=\left(1-\frac{\left(n-p_{1}\right) !\left(n-p_{2}\right) !}{n !\left(n-p_{1}-p_{2}\right) !}\right)$.

Now,

$$
\begin{aligned}
& \mathbb{E}\left[\tilde{\sigma}^{2}(R)\right]=\frac{1 /(1-x)}{\left(\rho_{n}^{e}\left(\begin{array}{l}
n \\
p
\end{array}\right)|I s o(R)|\right)^{2}} \mathbb{E}\left[\sum_{\substack{W \subseteq K_{n}: W=S \cup T, S, T \cong R,|S \cap T|=1, p}} \mathbf{1}(W \subseteq G)\right]-\frac{x \rho_{n}^{-2 e} \mathbb{E}\left[\hat{P}(R)^{2}\right]}{(1-x)} \\
& =\frac{1 /(1-x)}{\left(\rho_{n}^{e}\left(\begin{array}{l}
n \\
p
\end{array}\right)|I s o(R)|\right)^{2}} \mathbb{E}\left[\sum_{\substack{W \subseteq K_{n}: W=S \cup T, S, T \cong R,|S \cap T|=1, p}} \mathbf{1}(W \subseteq G)\right]-\frac{x \rho_{n}^{-2 e}\left[\operatorname{Var}(\hat{P}(R))+P(R)^{2}\right]}{(1-x)} \\
& =\frac{1}{1-x} \frac{1}{\left(\rho_{n}^{e}\left(\begin{array}{l}
n \\
p
\end{array}\right)|I s o(R)|\right)^{2}} \mathbb{E}\left[\sum_{\substack{W \subseteq K_{n}: W=S \cup T, S, T \cong R,|S \cap T|=1, p}} \mathbf{1}(W \subseteq G)\right]-\frac{x \tilde{P}(R)^{2}}{1-x} \\
& -\frac{x \rho_{n}^{-2 e} \operatorname{Var}(\hat{P}(R))}{(1-x)} \\
& =\frac{1}{1-x} \frac{1}{\left(\rho^{e}\left(\begin{array}{l}
n \\
p
\end{array}\right)|I s o(R)|\right)^{2}} \sum_{\substack{W: W=S \cup T \\
S, T \cong R, S \cap T \neq \emptyset}} \mathbb{E}\left[\sum_{W \subseteq K_{n}} \mathbf{1}(W \subseteq G)\right]-\frac{x \tilde{P}(R)^{2}}{1-x} \\
& -\frac{1}{(1-x)\left(\rho_{n}^{e}\left(\begin{array}{l}
n \\
p
\end{array}\right)|I s o(R)|\right)^{2}} \mathbb{E}\left[\sum_{\substack{W \subseteq K_{n}: W=S \cup T, S, T \cong R, 1<|S \cap T|<p}} \mathbf{1}(W \subseteq G)\right]-\frac{x \rho_{n}^{-2 e} \operatorname{Var}(\hat{P}(R))}{(1-x)} \\
& =\frac{\operatorname{Var}\left(\rho_{n}^{-e} \hat{P}(R)\right)}{(1-x)}-\frac{x \rho_{n}^{-2 e} \operatorname{Var}(\hat{P}(R))}{(1-x)}-o\left(\operatorname{Var}\left(\rho_{n}^{-e} \hat{P}(R)\right)\right) \\
& =\operatorname{Var}\left(\rho_{n}^{-e} \hat{P}(R)\right)-o\left(\operatorname{Var}\left(\rho_{n}^{-e} \hat{P}(R)\right)\right)
\end{aligned}
$$

Similarly, we get,

$\mathbb{E}\left[\tilde{\sigma}\left(R_{1}, R_{2}\right)\right]=\operatorname{Cov}\left(\rho_{n}^{-e_{1}} \hat{P}\left(R_{1}\right), \rho_{n}^{-e_{2}} \hat{P}\left(R_{2}\right)\right)-o\left(\operatorname{Cov}\left(\rho_{n}^{-e_{1}} \hat{P}\left(R_{1}\right), \rho_{n}^{-e_{2}} \hat{P}\left(R_{2}\right)\right)\right)$

Now, from the Theorem 1(a) in [1], we know that as $\lambda_{n} \rightarrow \infty$, if $\hat{\rho}_{n}=\frac{\bar{D}}{n-1}$ as defined in (2.6),

$$
\frac{\hat{\rho}_{n}}{\rho_{n}} \stackrel{P}{\rightarrow} 1
$$

So, using the estimate $\hat{\rho}_{n}$, we get that,

$$
\frac{\hat{\sigma}^{2}(R)}{\sigma^{2}(R ; \rho)} \stackrel{P}{\rightarrow} 1, \quad \frac{\hat{\sigma}\left(R_{1}, R_{2}\right)}{\sigma\left(R_{1}, R_{2} ; \rho\right)} \stackrel{P}{\rightarrow} 1
$$


B3. Proof of Lemma 8. Given $G$,

$$
\begin{aligned}
& \mathbb{E}\left[\hat{\sigma}_{B i}^{2}(R) \mid G\right] \\
= & \sum_{\substack{W=S \cup T, S, T \cong R,|S \cap T|=1, p}} \frac{\left(\hat{\rho}_{n}^{e}{ }_{W}\left(\begin{array}{c}
n \\
p_{W}
\end{array}\right)|I s o(R)|\right)}{(1-x)\left(\hat{\rho}_{n}^{e}\left(\begin{array}{c}
n \\
p
\end{array}\right)|I s o(R)|\right)^{2}} \mathbb{E}\left[\bar{P}_{B i}(W) \mid G\right]-\frac{x \mathbb{E}\left[\hat{\rho}_{n}^{-2 e} \bar{P}_{B i}(R)^{2} \mid G\right]}{(1-x)} \\
= & \sum_{\substack{W=S \cup T, S, T \cong R,|S \cap T|=1, p}} \frac{\left(\hat{\rho}_{n}^{e} e_{\left.\left(\begin{array}{c}
n \\
p_{W}
\end{array}\right)|I s o(R)|\right)}^{(1-x)\left(\hat{\rho}_{n}^{e}\left(\begin{array}{l}
n \\
p
\end{array}\right)|I s o(R)|\right)^{2}} \hat{P}(W)-\frac{x \hat{\rho}_{n}^{-2 e} \hat{P}(R)^{2}}{(1-x)}-\frac{x \operatorname{Var}\left(\hat{T}_{B i}(R)\right)}{1-x}\right.}{=\hat{\sigma}^{2}(R)-\frac{x \operatorname{Var}\left(\hat{T}_{B i}(R)\right)}{1-x}=\hat{\sigma}^{2}(R)-o\left(\hat{\sigma}^{2}(R)\right)}
\end{aligned}
$$

where the last inequality follows since $x=O\left(\frac{1}{n}\right)$ and Theorem 3.1 and Theorem 3.2 for $i=1,2$.

Similarly, we get,

$$
\mathbb{E}\left[\hat{\sigma}_{B i}\left(R_{1}, R_{2}\right) \mid G\right]=\hat{\sigma}\left(R_{1}, R_{2}\right)-o\left(\hat{\sigma}^{2}(R)\right)
$$

So using Lemma 7, we have that,

$$
\frac{\hat{\sigma}_{B i}^{2}(R)}{\sigma^{2}(R ; \rho)} \stackrel{P}{\rightarrow} 1, \quad \frac{\hat{\sigma}_{B i}\left(R_{1}, R_{2}\right)}{\sigma\left(R_{1}, R_{2} ; \rho\right)} \stackrel{P}{\rightarrow} 1 \quad \text { for } i=1,2
$$

\section{References.}

[1] Bickel, P. J., Chen, A. and Levina, E. (2011). The method of moments and degree distributions for network models. Ann. Statist. 39 2280-2301. . MR2906868

[2] Thompson, S. K. (2012). Sampling, third ed. Wiley Series in Probability and Statistics. John Wiley \& Sons Inc., Hoboken, NJ. . MR2894042 (2012k:62024)

Department of Statistics

44 Kidder Hall

Corvallis, OR, 97331

E-MAIL: bhattash@science.oregonstate.edu
Department of Statistics 367 Evans Hall

Berkeley, CA, 94720

E-MAIL: bickel@stat.berkeley.edu 